\title{
Effects of Hydrostatic Pocket Shape on the Flow Pattern and Pressure Distribution
}

\author{
M. J. BRAUN and M. DZODZO \\ Department of Mechanical Engineering, University of Akron, Akron, OH 44325
}

\begin{abstract}
The flow in a hydrostatic pocket is numerically simulated using a dimensionless formulation of the 2-D Navier-Stokes equations written in primitive variables, for a body fitted coordinates system, and applied through a collocated grid. In essence, we continue the work of Braun et al. [1993a,1993b] and extend it to the study of the effects of the pocket geometric format on the flow pattern and pressure distribution. The model includes the coupling between the pocket flow and a finite length feedline flow, on one hand, and the pocket and its adjacent lands on the other hand. In this context we shall present, on a comparative basis, the flow and the pressure patterns at the runner surface for square, ramped-Rayleigh step, and arc of circle pockets. Geometrically all pockets have the same footprint, same lands length, and same capillary feedline. The numerical simulation uses the Reynolds number based on the lid(runner) velocity and the inlet jet strength $\mathbf{F}$ as the dynamic similarity parameters. The study aims at establishing criteria for the optimization of the pocket geometry in the larger context of the performance of a hydrostatic bearing.
\end{abstract}

Key Words: Hydrostatic pocket; Numerical; Flow pattern; Pressure; Navier-Stokes

$\mathbf{T}$ he problem of the flow structure in a closed, lid driven cavity has been treated extensively in the last two decades. As a part of the class of separated flows, the closed cavity problem had principally a fundamental theoretical importance.

Brandeis and Rom [1980] solved the open driven cavity flow field where the driving factor is a shear layer similar to that presented by O'Brien [1982]. The global flow field which contained shear and recirculating flows is solved iteratively by matching the boundary conditions between the two flows. Kumar and Yuan [1989] solved the problem of the penetration of a vertical downward jet in a rectangular fully enclosed cavity. The authors found that the strength of the jet and the cavity aspect ratio (depth to width) played a major role in shaping the flow recirculation structure. Most importantly it was concluded that as the cavity becomes deeper a constant intensity jet looses its ability to penetrate deeply, and thus greatly affects the nature of the recirculation pattern, and interaction with the shear layer.
In the recent years there has been a sustained activity towards extending this work to the geometry and purposes of the hydrostatic pocket. Most of the recent work concentrated towards the implicit treatment of the pocket performance in the numerical environment of the total solution of the Reynolds equation for the entire journal hydrostatic bearing. A rather complete review of this literature is presented by Braun et al. [1993a] in his study of the steady state flow structure and pressure patterns in a hydrostatic cavity of variable cross section.

Numerous authors have analyzed the overall thermofluid and dynamic performance of the hydrostatic bearing. Thus, Artiles et al. [1982], Castelli and Shapiro [1967], Redecliffe and Vohr [1969], Belousov [1976], Braun and Wheeler [1987], San Andres [1990], Goryunov and Belousov [1969], Davies and Leonard [1970], have presented analytical and numerical analyses of the pressures and/or dynamic coefficients development in multi-pocketed hydrostatic journal bearing(HJB). A second group of researchers performed overall experi- 
mental studies concerning the flow characteristics, frictional characteristics, pressures, and dynamics of hydrostatic bearings. In this context one can mention Shinkle and Hornung [1965], Leonard and Davies [1971], Ho and Chen [1979, 1980, 1984], Aoyama et al. [1982], Chaomleffel and Nicholas [1986], Scharrer et al. [1991a, b], Spica et al. [1986]. A third group of investigators presented comparisons between their analytical/ numerical models and their own experiments for the thermofluid and dynamic behavior of the HJB [Heller, 1974, Betts and Roberts, 1969, Bou-Said and Chaomleffel, 1988, Hunt and Ahmed, 1968]. Finally, a fourth group of researchers have concentrated on the mechanics particular to the flow in cavities related to step bearings, spiral seal grooves and HJB. Ettles [1968] gave an insightful analysis of the flow in a bearing groove without hydrostatic effects, while Heckelman and Ettles [1987] studied the viscous and inertial pressure effects at the inlet of a bearing cavity film. Pan [1974], Constantinescu and Galetuse [1976], Tichy and Bourgin [1985] also looked at pressure entrance effects at the inlet of a bearing film. Zuk and Renkel [1971] analyzed the spiral groove pumping seal using the lid driven cavity model, but did not consider the leakage rates at the top of the cavity since the top of the cavity was closed. Recent work of Braun [1990] and Braun and Batur [1991] contained flow visualization experiments detailing fundamentals of the flow and the pressure patterns both in the pocket of a simulated HJB, and on the contiguous lands. Using fluid tracers the authors evidenced the hydrostatic jet penetration, the formation of the secondary eddies both upstream and downstream of the main jet, and the fluid turn around zone(TAZ) in the upstream portion of the pocket land. This TAZ becomes particularly dominant as the velocity of the shaft increases. In the limit, it was found, no jet borne fluid exits at the upstream end of the pocket, and only a thin layer of fluid attached to the rotating shaft is carried through the control surface of the cavity. Finally, San Andres and Velthuis [1991], and Braun et al. [1993a] developed steady state codes for the simulation of the laminar flow in the pockets of an HJB. Both authors confirmed that the pressure in the recess is not uniform, and inertia induced pressure drops occur at the pocket edges when the fluid flows outward. Braun [1993a, b] has also showed that for pockets with aspect ratios between $0.2<$ pocket depth/ pocket length $<1$, an increase in the shaft angular velocity causes the pressure in the upstream portion of the pocket to decrease, followed by a process of recovery in the central region, and a sharp increase in the downstream(exit) region of the pocket. These phenom- ena are apparently due to a combination of backstep and Rayleigh step type flows.

\section{SCOPE OF WORK}

The work reviewed above deals with the flow in open cavities driven by shear layers, in a cavity with a penetrating jet or with the overall experimental and analytical behavior of hydrostatic bearings. The work presented in this paper specifically extends the work of San Andres and Velthuis [1991] and Braun et al. [1993a,b], to hydrostatic pockets of variable non-cartesian geometry. The jet feedline, the pocket and the adjacent lands are modeled in a synthesis configuration that has direct pertinence to the overall hydrostatic journal bearing performance. The clearance was kept constant in size, and its magnitude was similar to the ones found in a functional hydrostatic bearing. The jet penetration, the interaction of the shear layer with the cavity flow, the presence of the lands and their geometry, all affect the flow patterns, as well as the magnitude, profile, and the trends of the pressures and velocities in the pocket.

\section{FORMULATION OF THE MATHEMATICAL MODEL}

\section{Geometry}

The geometry of three typical shapes of hydrostatic pockets are considered in this analysis. They are presented in Figs. 1A, 1B, 1C. The physical dimensions are tabulated in Table I. All the pockets are outfitted at the bottom with the same capillary feedline of length, $L_{2}$ and diameter $B$. All three pockets have the same projected footprint of length $L$ and the length of the lands is $M$. The main physical parameter of the square pocket of Fig. 1A, is its aspect ratio, $L / D$. The circular shaped pocket of Fig. $1 \mathrm{~B}$, represents an arc of circle of radius $R$. The pocket is defined by the same depth $D$ and length $L$ as the square pocket. Figure 1C, depicts the ramped Rayleigh pocket that is characterized by the same maximum depth $D$, and length $L$, as the other two pockets, but also by the angle of the $\operatorname{ramp}, \alpha=\operatorname{arctg}[D /(L-B)]$. All three geometries introduce the assumption that the cavity width is much larger than its length or depth. Thus the problem reduces to a two dimensional case, in coordinates $\left(Y_{1}, Y_{2}\right)$. The process is steady-state, and the fluid is Newtonian and incompressible. The properties of the fluid $(p, v)$ are kept constant. 


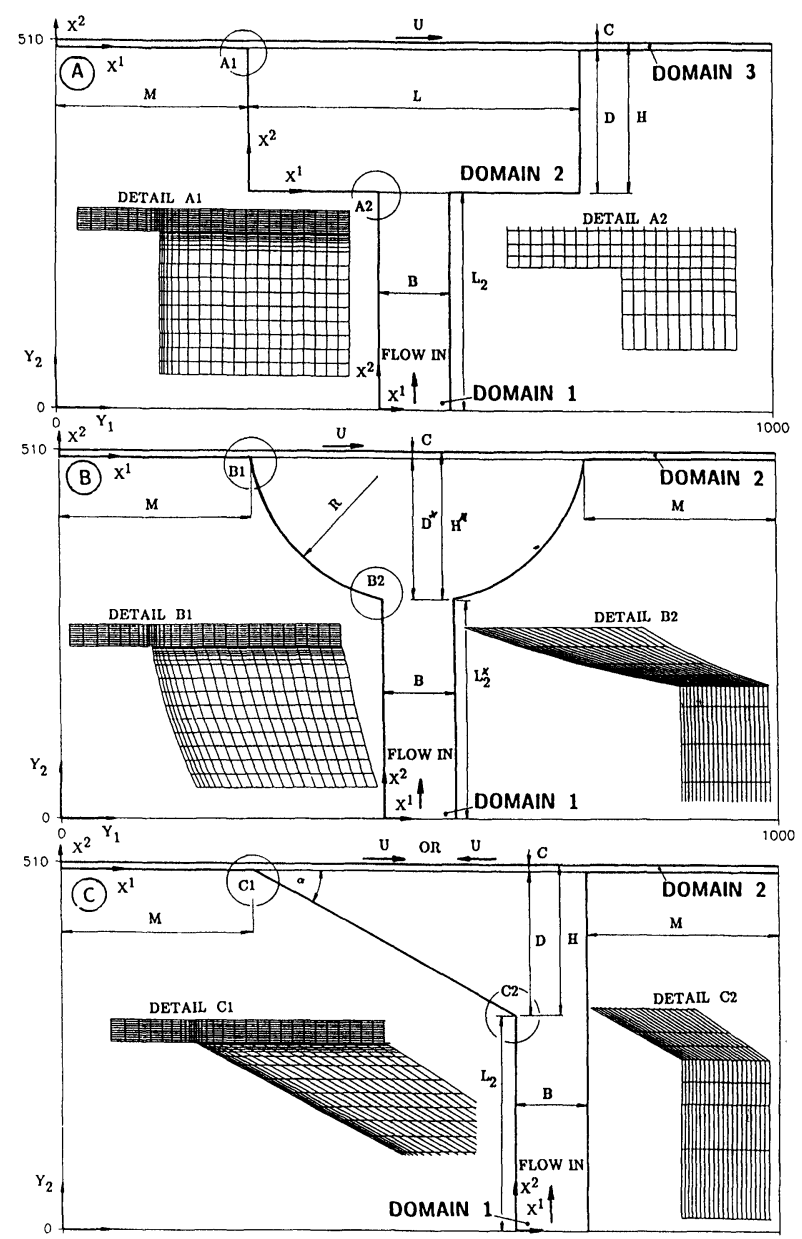

FIGURE 1 Geometry of the square, circular, and ramped-Rayleigh pockets.

Dimensionless equations for the fluid model. To obtain a certain degree of generality in the discussion of the

TABLE I

The dimensions of the considered pockets

\begin{tabular}{llll}
\hline & dimensionless & inches & \multicolumn{1}{c}{ meters } \\
\hline $\mathrm{L}$ & 464.758 & 0.6971 & 0.01770728 \\
$\mathrm{M}$ & 267.621 & 0.4149 & 0.01053926 \\
$\mathrm{D}$ & 200 & 0.3 & 0.00762 \\
$\mathrm{D}^{*}$ & 194.62 & 0.2919 & 0.007415 \\
$\mathrm{C}$ & 10 & 0.015 & 0.000381 \\
$\mathrm{H}$ & 210 & 0.315 & 0.008001 \\
$\mathrm{H}^{*}$ & 204.62 & 0.30693 & 0.007796 \\
$\mathrm{~B}$ & 100 & 0.15 & 0.00381 \\
$\mathrm{~L}_{2}$ & 300 & 0.45 & 0.01143 \\
$\mathrm{~L}_{2}$ & 305.38 & 0.4581 & 0.011635 \\
$\mathrm{R}^{*}$ & 35 & 0.3525 & 0.0089535 \\
$\mathrm{R}_{\mathrm{s}}$ & 1000 & 1.5 & 0.0381 \\
$\mathrm{~h}=\mathrm{R}_{\mathrm{s}} / 1000$ & 1 & 0.0015 & $3.81 * 10^{-5}$ \\
\hline
\end{tabular}

results, the governing equations of motion are cast in a dimensionless form. The characteristic length has been chosen to be $h=R_{s} / 1000$. The equations are written for a non-orthogonal body fitted coordinate system in accordance with Peric [1985] formulation. The collocated control volume cells and the corresponding local arbitrary coordinates $X^{1}, X^{2}$ are presented in Fig. 1 (Details $\mathrm{A} 1, \mathrm{~A} 2, \mathrm{~B} 1, \mathrm{~B} 2, \mathrm{C} 1$ and $\mathrm{C} 2$ ).

Using these notations, and the dimensionless variables defined in the nomenclature, the continuity equation takes the form

$\frac{\partial}{\partial X^{j}}\left(U_{k} \beta_{k}^{j}\right)=\frac{\partial \boldsymbol{U}_{1}}{\partial X^{1}}+\frac{\partial \boldsymbol{U}_{2}}{\partial X^{2}}=0$, where $\boldsymbol{U}_{j}=U_{1} \boldsymbol{\beta}_{1}^{j}+U_{2} \boldsymbol{\beta}_{2}^{j}$

The momentum equations for Cartesian velocity components $U_{i}(i=1,2)$ can be written in contracted form as

$\frac{\partial}{\partial X^{j}}\left[\boldsymbol{U}_{j} U_{i}-\frac{1}{J}\left(\frac{\partial U_{i}}{\partial X^{m}} \beta_{k}^{j} \beta_{k}^{m}+\frac{\partial U_{k}}{\partial X^{m}} \beta_{i}^{m} \boldsymbol{\beta}_{k}^{j}\right)+P \beta_{i}^{j}\right]=0$

where $\beta_{j}^{i}$, represents the cofactor of the $i$ th row and the $j$ th column in the Jacobian matrix $\boldsymbol{J}$ of the coordinate transformation

$$
y^{i}=y^{i}\left(x^{j}\right)
$$

where $y^{i}$ represents the $Y_{1}, Y_{2}$ reference Cartesian system of coordinates. For the two dimensional application of the Navier-Stokes equation, the cofactors are

$$
\beta_{1}^{1}=\frac{\partial Y_{2}}{\partial X^{2}}, \beta_{2}^{1}=-\frac{\partial Y_{1}}{\partial X^{2}}, \beta_{1}^{2}=-\frac{\partial Y_{2}}{\partial X^{1}}, \beta_{2}^{2}=\frac{\partial Y_{1}}{\partial X^{1}}
$$

The advantage of this formulation resides in its natural ease to interface with the irregular geometry of the pockets of Figs. 1B and 1C. For the rectangular pocket the computational domain was split into three domains (Fig. 1A), while for the irregular geometry (circular and triangular) pockets in two domains (Fig. 1B and 1C) respectively. For each domain local non-orthogonal coordinates $\left(X^{1}, X^{2}\right)$ were used.

\section{Boundary Conditions}

The boundary conditions on the lands at the cavity open ends are:

$$
\begin{aligned}
& \frac{\partial U_{1}}{\partial Y_{1}}=0 \text { and } \frac{\partial U_{2}}{\partial Y_{1}}=0 \text { where } Y_{1}=0 \text { and } \\
& Y_{1}=M+L+M=1000, \text { for } 500 \leq Y_{2} \leq 510
\end{aligned}
$$


The numerical values in Eq. 5 represent non-dimensional coordinates of the pocket's geometry. The pressure on the boundaries was set to a reference environment pressure, $P=0$. The same pressure conditions $(P=0)$ applied on the land's inlet and exit at $Y_{1}=0$ and $Y_{1}=$ $M+L+M=1000$, and for $500 \leq Y_{2} \leq 510$. All other pressures inside the domain were calculated taking the boundary pressures as the reference pressure. Using these boundary pressures, a symmetrical mass outflow at both ends was obtained for the case when the runner is stationary. Similarly to Shyy's [1985] model, our model did not need to prescribe the lands' mass outflow ratio.

In the entrance region where the fully developed, perpendicular hydrostatic jet, enters the capillary feedline, one can write

$$
\begin{aligned}
& U_{2}=-F\left[Y_{1}-\left(S-\frac{B}{2}\right)\right]\left[Y_{1}-\left(S+\frac{B}{2}\right)\right] \\
& \text { where } Y_{2}=0 \text { and } S-\frac{B}{2} \leq Y_{1} \leq S+\frac{B}{2}
\end{aligned}
$$

where $S=M+L / 2=500$ for the rectangular and circular pocket and $S=M+L-B / 2=682.379$ for the triangular pocket. The strength of the jet was varied by changing the dimensionless parameter $\boldsymbol{F}=\frac{1}{2}\left(\frac{\partial P}{\partial Y_{2}}\right)$ to different values $\left(\boldsymbol{F}=10^{-4}, F=2 * 10^{-4}\right.$ and $\left.\boldsymbol{F}=4^{*} 10^{-4}\right)$.

The boundary conditions at the driving lid are:

$$
\begin{aligned}
& U_{I}=\operatorname{Re} \text { and } U_{2}=0 \\
& \text { where } Y_{2}=510,0 \leq Y_{1} \leq M+L+M
\end{aligned}
$$

During the parametric study, the horizontal velocity of the runner $U_{1}$, is set to coincide with $\boldsymbol{R} \boldsymbol{e}=1,2,4$, and 8 . The rest of the boundary conditions set the magnitudes of the velocities to $U_{1}=0$ and $U_{2}=0$ along the walls. For the various computational domains (see Fig. 1) the values calculated at the boundaries of the contiguous domains were used as boundary conditions. This procedure is similar to the 'slab by slab' method of calculation used by Pratap and Spalding [1976] for staggered grids. For the work presented here the procedure has been adapted to collocated cells in the same manner as described by Dzodzo [1991].

\section{THE NUMERICAL PROCEDURE}

A finite volume method based on the finite difference method was applied for the numerical implementation of
Eqs. 1 to 7. The method used non-orthogonal coordinates and a collocated grid [Peric,1985] The SIMPLE procedure [Patankar and Spalding, 1972, and Patankar, 1980] was used for solving the resulting set of equations.

Figures 1A, 1B and 1C present in details A1, B1, C1 parts of the discrete grid superimposed on the cavity. For the rectangular pocket (Fig. 1A) the computational domains $(1,2$, and 3$)$ contained $20 \times 20,100 \times 40$ and $200 \times 10$ control cells, respectively. For the nonorthogonal pockets $100 \times 60$ and $200 \times 10$ control cells were used for domains 1 and 2, respectively.

The convergence criteria was set through the calculation of the overall average dimensionless mass imbalance, $S_{m}$, for each of the component domains. This criteria can be expressed as

$S_{m}=\left(\sum\left|\boldsymbol{U}_{1 w^{\prime}} A_{W^{-}} \boldsymbol{U}_{1 e} A_{e}+\boldsymbol{U}_{2 s} A_{s^{-}} \boldsymbol{U}_{2 n} A_{n}\right|\right) \frac{1}{n}<10^{-4}(8)$

Using $S_{m}<10^{-4}$, translates in a difference, between all dimensionless mass inflow and all outflow over the summed domains, of less than $3.5 \%$.

\section{RESULTS AND DISCUSSION}

As mentioned before the main thrust of this study is a comparative analysis of the flow and pressure patterns in the geometries of the pockets shown in Figs. 1. The numerical experiments were run parametrically as a function of the Reynolds number $(\boldsymbol{R} \boldsymbol{e}=1,2,4,8)$ and the jet strength, $\left(\boldsymbol{F}=1 * 10^{-4}, 2 * 10^{-4}, 4 * 10^{-4}\right)$. One can get a feeling as to the physical magnitudes of these variables from an inspection of Table II.

\section{Flow in the Square Pocket}

Figure 2 presents in a matrix format the effects of the said parameters on the steady state flow patterns. The effect of the increase in $\boldsymbol{R} \boldsymbol{e}$ can be followed row-wise, while the jet strength increases along the columns of the figure. The velocity profiles at the inlet $\left(Y_{1}=0\right)$, and exit $\left(Y_{1}=\right.$ 1000) of the pockets lands are shown in the DETAIL figures that accompany every pocket case. In Figure 2A1 the jet has its lowest strength and it appears that the flow is dominated by the shear layer effects (and the magnitude of the $\boldsymbol{R} \boldsymbol{e}$ number). Thus, in the pocket's upstream region, there is a relatively large Turn Around Zone (TAZ), and one can see in the DETAIL A1I, that the exit upstream velocities are quite small. On further inspection, one can see a large developed Main Vortical Cell (MVC), and a small Upstream Secondary Eddy (USE). As the jet strength increases, Figs. 2A2, and 2A3, it starts 
TABLE II

The runner velocities and mean feeding jet velocities as functions of $R e$ and $F$ parameters, respectively (for $\rho=900 \mathrm{~kg} / \mathrm{m}^{3}, \mu=9 * 10^{-2} \mathrm{~kg} / \mathrm{ms}$ $\left(\nu=\mu / \rho=100 * 10^{-6} \mathrm{~m}^{2} / \mathrm{s}\right)$ and $R_{s}=1.5 \mathrm{in}=38.1 \mathrm{~mm}, h=R_{s}$ ' $1000=38.1 * 10^{-6} \mathrm{~m}$ )

\begin{tabular}{lccccc}
\hline $\boldsymbol{R} \boldsymbol{e}=U$ & & 1 & 2 & 4 & 8 \\
\hline linear speed & $\mathrm{m} / \mathrm{s}$ & 2.625 & 5.250 & 10.5 & 21.0 \\
rot. speed & $\mathrm{rpm}$ & 658 & 1315.8 & 2631.7 & 5263.4 \\
\hline $\boldsymbol{F}$ & & $1 * 10^{-4}$ & $2 * 10^{-4}$ & $4 * 10^{-4}$ & \\
\hline
\end{tabular}

average jet

\begin{tabular}{lllll} 
inlet velocity $\quad \mathrm{m} / \mathrm{s}$ & 0.4374 & 0.8749 & 1.7498 \\
\hline
\end{tabular}

to dominate the interaction between the jet and the shear layer, with a consequent decrease in the TAZ, and an increase of the exit fluid velocity in the upstream region (DETAIL A3I). At the same time as the USE increases, the center of the MVC is pushed downward and leftward (away from the downstream exit). From the profiles of the exit velocities it is clear that the jet strength domination is associated with a strong Poiseuille action on the lands. As the Reynolds number increases to 4 and 8 respectively (Figs. 2B and 2C), there is a gradual growth of the TAZ as well as an increased domination of the Couette flow, both on the upstream and downstream lands of the pockets (see DETAILS B1I through B3I, C1I through $\mathrm{C} 3 \mathrm{I}$, for the upstream inlet region, and $\mathrm{B} 1 \mathrm{E}$ through $\mathrm{B} 3 \mathrm{E}$, and $\mathrm{C} 1 \mathrm{E}$ through $\mathrm{C} 3 \mathrm{E}$, for the downstream exit region). The MVC has now moved rightward and it even deforms slightly to an ovoid shape, Fig. 2C3.

\section{Flow in the Circular Pocket}

The $\boldsymbol{R} \boldsymbol{e}$ and $\boldsymbol{F}$ parameters were set at magnitude levels equal to the ones used in the numerical experiments shown in Figs. 2. Upon analysis of both the qualitative and quantitative data of Figs. 3, it has been concluded that the same physical flow characteristics observed in Figs. 2 are present in Figs. 3. The only difference, is the generalization of the ovoid shape of both the MVC and the USE. It is apparent that square and circular pockets having the same footprint and maximum depth exhibit in general the same flow and velocity characteristics.

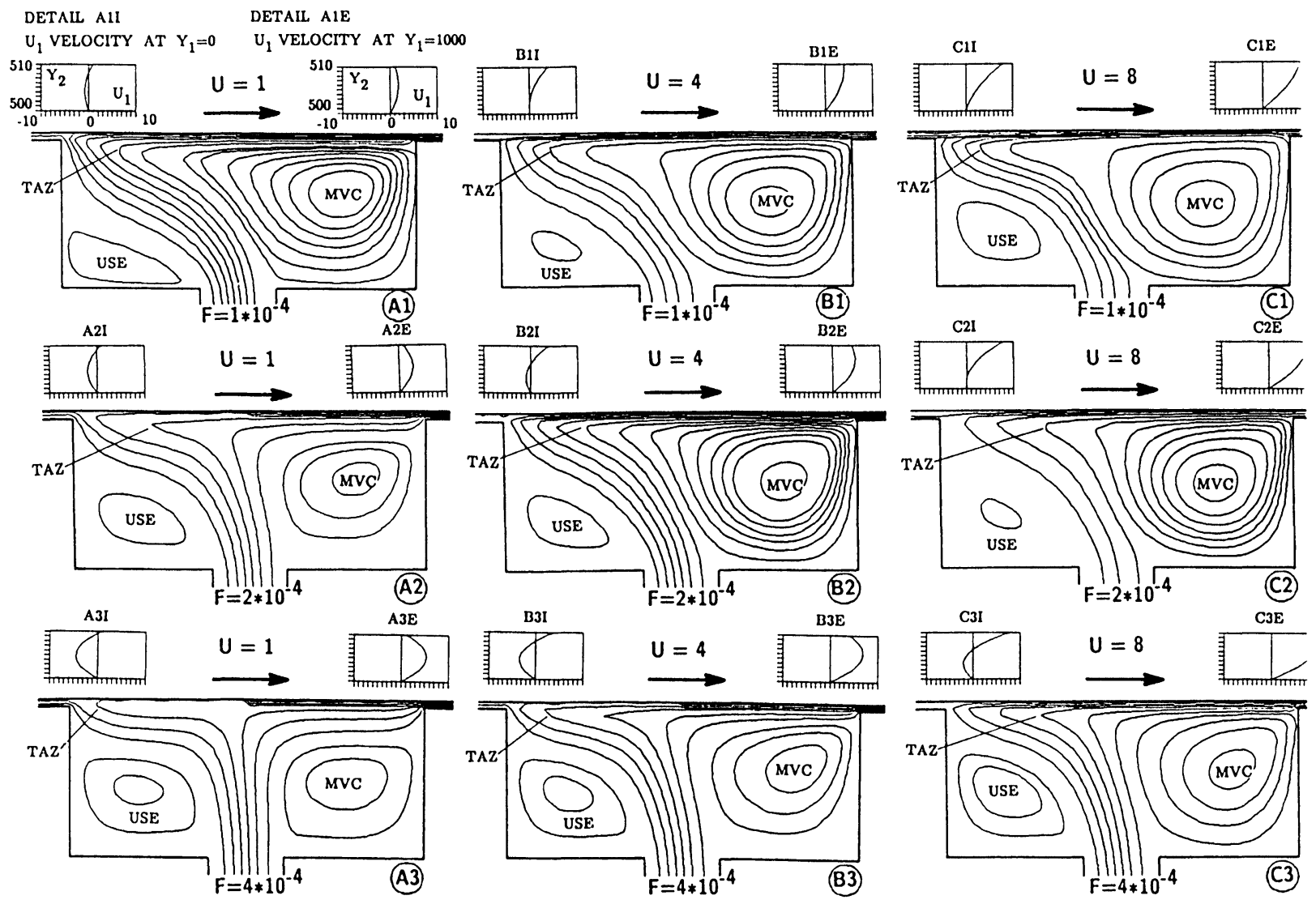

FIGURE 2 Flow patterns in the square pocket for variable $\boldsymbol{R} \boldsymbol{e}(=1,4,8)$ and $\boldsymbol{F}\left(=1 * 10^{-4}, 2 * 10^{-4}, 4 * 10^{-4}\right)$ numbers. 


\section{Flow in the Ramped-Rayleigh Step Pocket.}

Figures 4 and 5 present the flow patterns when the runner moves leftward towards the $\operatorname{ramp}(U=-1,-4,-8)$, and away from the ramp (rightward, $U=1,4,8$ ), respectively.

During the leftward motion of the lid, Figs. 4, one can ascertain the universal existence of the MVC and disappearance of the USE, for all $\boldsymbol{R} \boldsymbol{e}$ and $\boldsymbol{F}$. The increase in the jet strength, Figs. 4A1, 4A2, 4A3, contributes to a downward motion and decrease in size of the MVC core. The explanation resides in the magnitude of the exit velocities shown in DETAILS A1E through A3E. When the exit velocity is small ( $\boldsymbol{F}$ is small), the runner shear layer dominates (A1E), and most of the fluid in the cavity recirculates in the MVC. As $\boldsymbol{F}$ increases, the Poiseuille effect start to dominate on the lands (A3E), the exit flow becomes considerable, the MVC gets smaller, and is displaced downward. As the Reynolds number increases to its maximum value (Figs. 4C1, 4C2, and 4C3), the shear layer effect (Couette effect) starts to dominate again (Fig. 4C1), pulling the MVC upward, and towards the downstream exit land. As $\boldsymbol{F}$ is further increased (Figs. 4C2, 4C3), a balance is struck between the Couette and Poiseuille effects. The MVC remains located in the downstream exit zone, but diminishes in size.

For the same geometry, but with a rightward motion of the runner, Fig. 5, some interesting differences appear in the flow patterns, when compared against Fig. 4. Generally, one can observe the formation and universal existence of an MVC that is close in nature to the MVC exhibited by the square pocket of Figs. 2. The USE appears and disappears as a function of the momentary interplay between $\boldsymbol{F}$ and $\boldsymbol{R} \boldsymbol{e}$. Thus, when $\boldsymbol{R} \boldsymbol{e}=1(U=1)$, and $\boldsymbol{F}=1 * 10^{-4}$ (Fig. 5A1), the land exit velocities, both upstream and downstream, are small, and most of the flow gets recirculated within a well developed MVC. A very large TAZ envelopes the MVC, which becomes a 'de facto' TAZ core. As the jet strength increases, the MVC gets eliminated, and a USE zone appears and starts to grow (Figs. 5A2, and 5A3). As the Reynolds number increases (Figs. 5B1, and 5C1) the MVC core becomes well established, keeps growing, and practically pushes

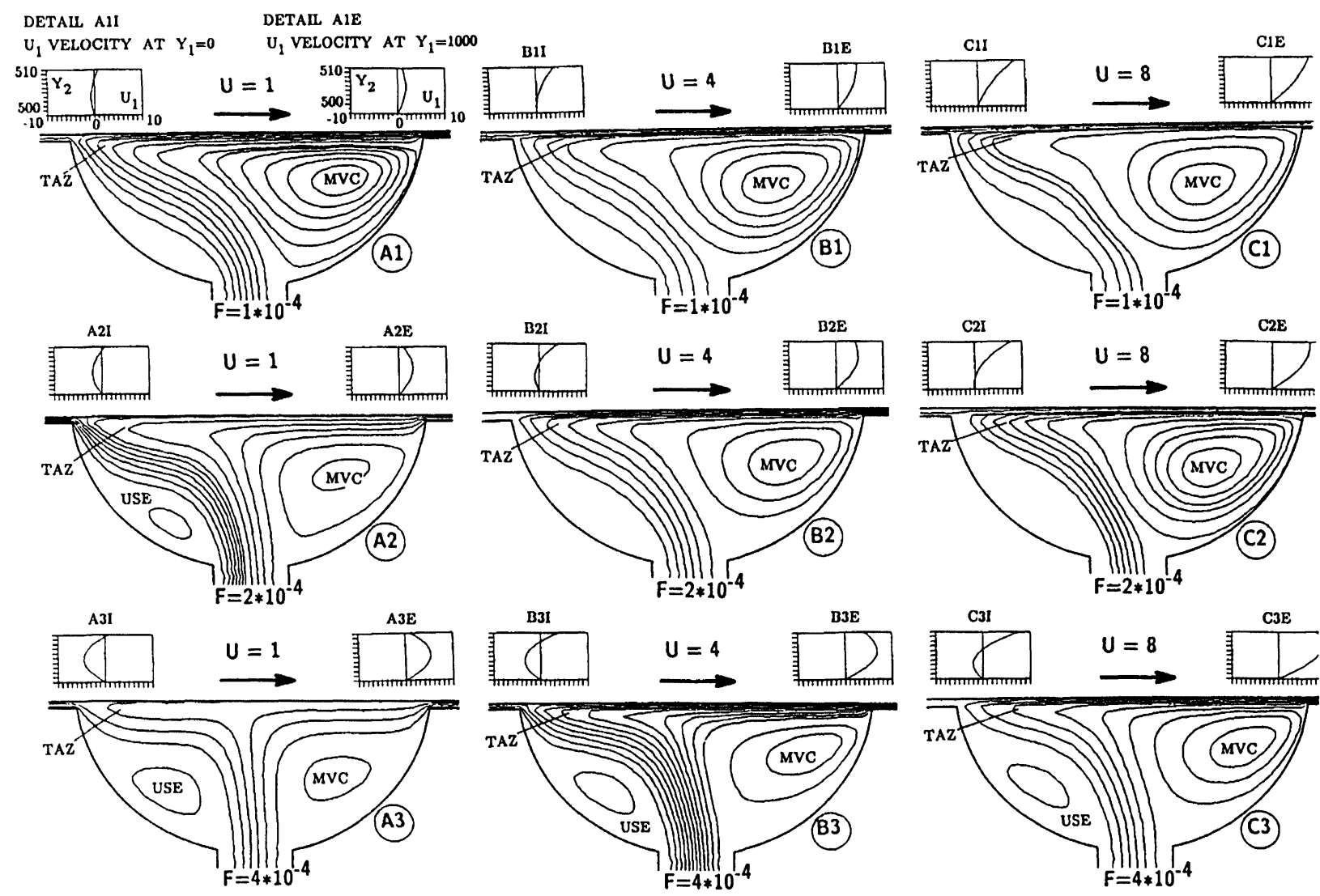

FIGURE 3 Flow patterns in the circular pocket for variable $\boldsymbol{R} \boldsymbol{e}(=1,4,8)$ and $\mathbf{F}\left(=1 * 10^{-4}, 2 * 10^{-4}, 4 * 10^{-4}\right)$ numbers. 


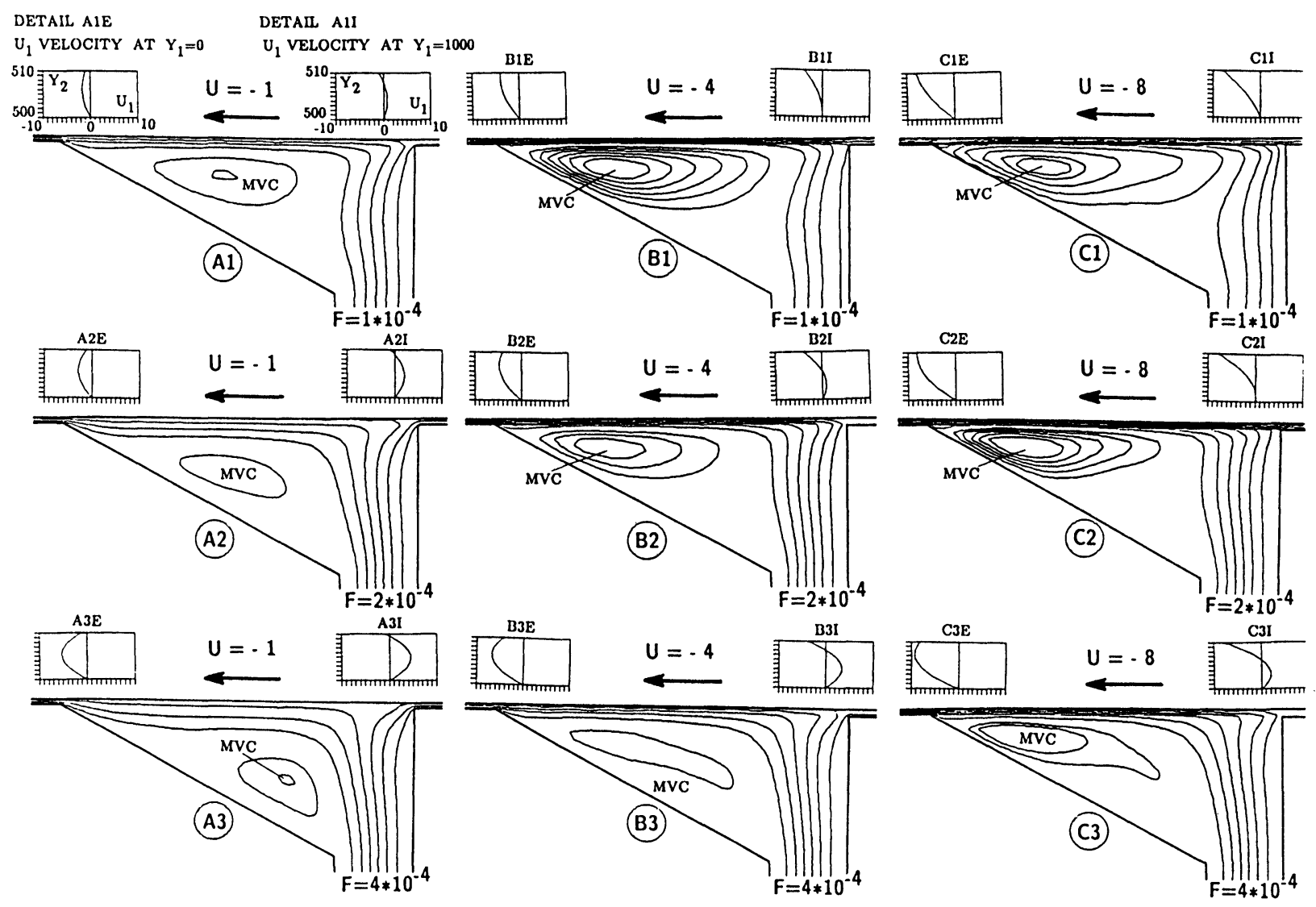

FIGURE 4 Flow patterns in the ramped-Rayleigh pocket for variable $\boldsymbol{R} \boldsymbol{e}(=1,4,8)$ and $\boldsymbol{F}\left(=1 * 10^{-4}, 2 * 10^{-4}, 4 * 10^{-4}\right)$ numbers, and right-to-left runner movement.

the USE out of existence. The subsequent jet strength increase (Figs. 5C2, and 5C3) contributes generally to the diminishing of the MVC and the reappearance of an incipient USE region.

\section{Pressure Developments}

Figures $6 \mathrm{~A}$ and $6 \mathrm{~B}$ present the pressure development in the square and the circular hydrostatic pockets, respectively. The jet strength is kept constant at $\boldsymbol{F}=1 * 10^{-4}$, while the Reynolds number is increased from 1 to 2,4 and 8 . With the increase in the $\boldsymbol{R e}$, both types of pockets experience the same gradual decrease in pressure in the backstep areas, and the spike increase in the exit Rayleigh step area. This phenomenon was reported, and explained earlier by Braun et al. [1993a]. It is noteworthy that the extent of the pressure depression zones $\mathrm{A}$ (Fig. 6A) and B (Fig. 6B) correlate well with the magnitude of the USE zones in Figs. $2 \mathrm{C} 1$ and $3 \mathrm{C} 1$ respectively. Thus the larger the USE is, the smaller the depression becomes. Besides this local phenomenon, there is little difference both in the overall qualitative and quantitative behavior of the pressures between the two pockets. This is consistent with the findings that the overall flow patterns of Figs. 2 and 3 also show little difference.

Figures 7A and 7B present the pressure profiles associated with the ramped-Rayleigh pockets as the runner moves leftward and rightward, respectively. As in Figs. 6 , the jet strength is kept constant at $\boldsymbol{F}=1 * 10^{-4}$, while the Reynolds number is increased from 1 to 2,4 and 8. One can again ascertain for both figures the general (and already discussed) phenomena of the pocket pressure drop with the increase in the velocity $(\boldsymbol{R} \boldsymbol{e})$ of the runner. The effects of the backstep and Rayleigh step are present as well. While the qualitative effects of the increase in Reynolds are the same between Figs. 7A and $7 \mathrm{~B}$, the magnitudes of both the pressure drops and pressure peaks are largely different. Thus, the pressure 


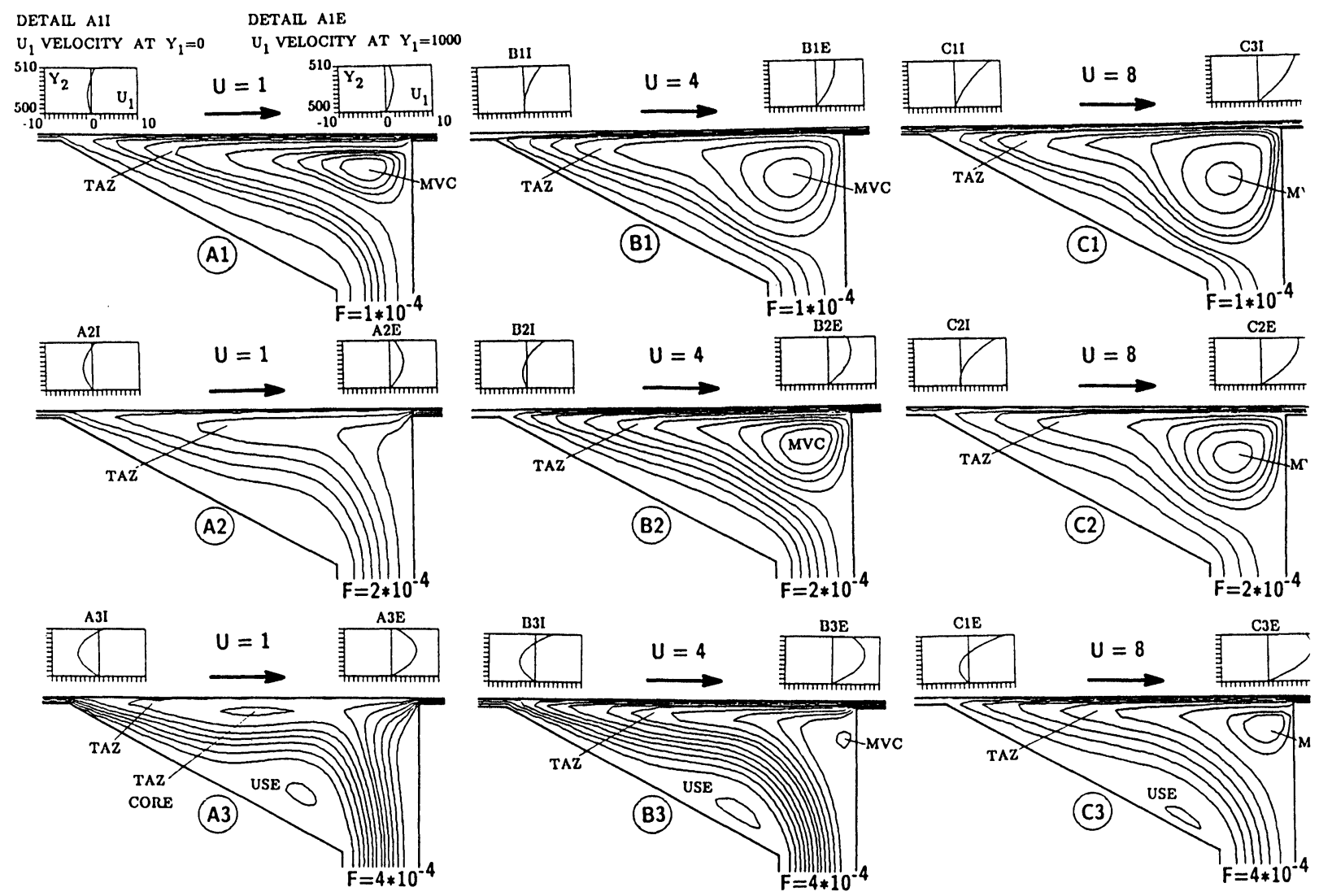

FIGURE 5 Flow patterns in the ramped-Rayleigh pocket for variable $\boldsymbol{R e}(=1,4,8)$ and $\boldsymbol{F}\left(=1 * 10^{-4}, 2 * 10^{-4}, 4 * 10^{-4}\right)$ numbers, and left-to-right runner movement.

drop in the pocket when the runner moves leftward, $\Delta \mathrm{p}_{1}$, is approximately twice the $\Delta p_{2}$ in the pocket when the runner moves rightward, Fig. 7B. Conversely, the pressure spike in Fig. 7A is twice as large as the pressure spike of Fig. 7B. It is apparent that both the pressure spikes and pressure drops are related to the size and position of the MVC in the pocket.

Figure 8 presents, on a comparative basis, the relationship between the pressures in all three types of pockets when $\boldsymbol{R} \boldsymbol{e}$ is kept constant $(\boldsymbol{R} \boldsymbol{e}=8)$, and the jet strength is varied from $\boldsymbol{F}=1 * 10^{-4}$, to $2 * 10^{-4}$, and $4 * 10^{-4}$, respectively. It is clear that the increase in $\boldsymbol{F}$ brings about a proportional increase in the pressure level in the pocket. At the same time it becomes evident that the spikes in pressure at the downstream exit of any of the pockets are more related to the variation in the Reynolds number, rather then to the strength of the jet. The spike as well as the pressure drop in the pocket, are thus very pronounced for the low $\boldsymbol{F}$ number cases when the physics are dominated by the velocity of the runner and the associated shear layer. For the high $\boldsymbol{F}$ number, the pressure spikes are almost eliminated due to the jet dominance.

\section{CONCLUSIONS}

This paper presents a study of the effects of pocket shape (not depth) on the flow patterns and the pressure magnitudes in a hydrostatic pocket. The flow is modeled by taking into consideration both the geometry of a portion of the feedline and the lands adjacent to the pocket. The laminar, two dimensional, steady-state, constant properties, Navier-Stokes equations are cast in a formulation that permits a body fitted coordinates numerical implementation. The results confirm the findings of Braun et $a l$. [1993a, b] and extend them to the shape of the pockets presented in this study. The pressure drops in the backstep region of the pocket and the pressure spike in the Rayleigh step portion are universally confirmed for the configurations covered in this study. It is shown that the flow patterns actually can explain these phenomena 

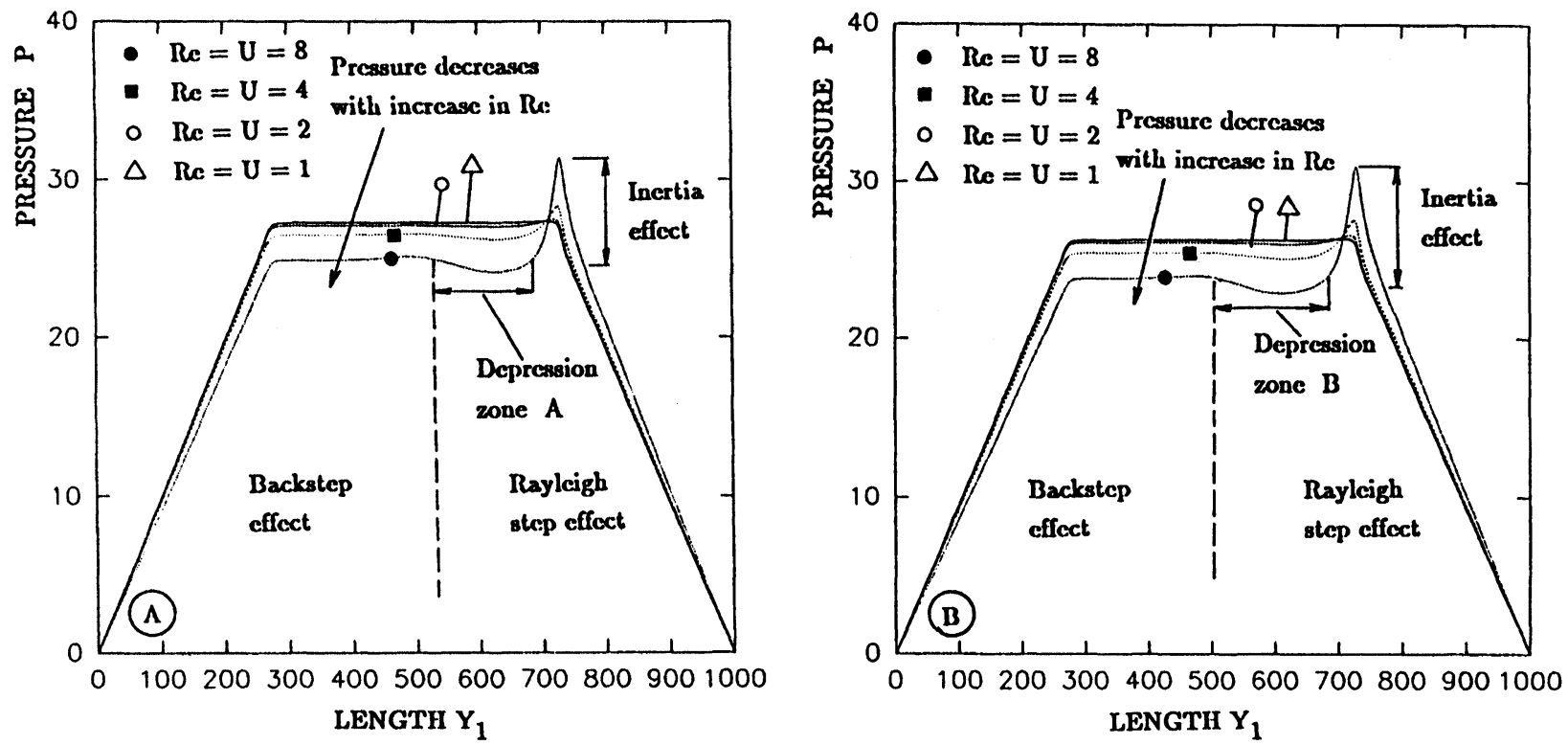

FIGURE 6 Pressure profiles development at constant jet strength $\boldsymbol{F}=1 * 10^{-4}$, and for $\boldsymbol{R e}(=1,2,4,8)$; A) the square pocket; B) the circular pocket

through the appearance and variation of the USE, MVC, and TAZ regions. The interplay and clear coupling between the Reynolds number and jet strength are clearly proven. It is shown that the pressure spikes at the pocket downstream exit are mainly related to the velocity of the runner (magnitude of the Reynolds number), and they diminish and disappear when the jet strength becomes dominant. The inertia effects appear mainly at the downstream exit of the pocket and are strictly related to the flow velocity in the area, regardless of whether they are
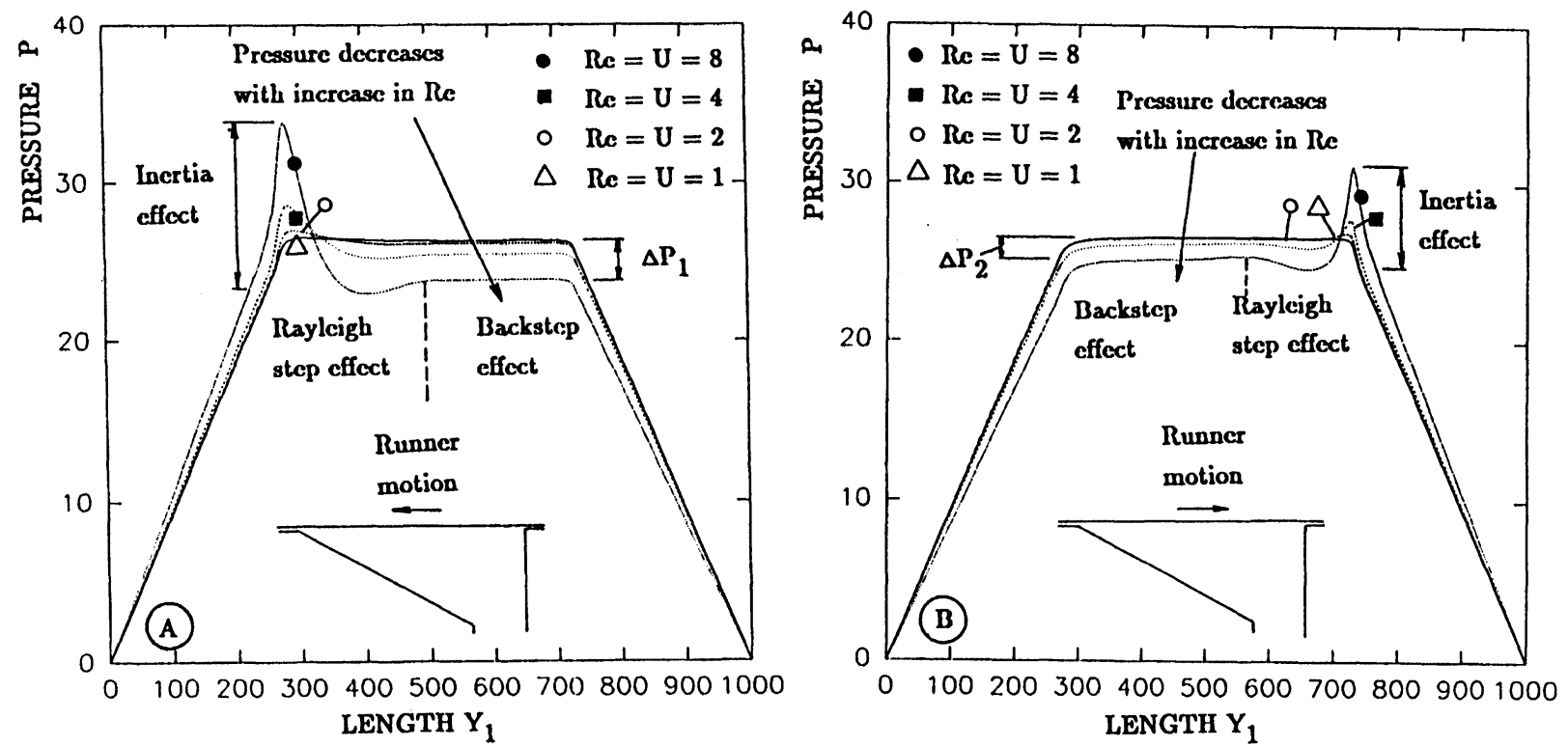

FIGURE 7 Flow patterns in the ramped-Rayleigh pocket at constant jet strength $\boldsymbol{F}=1 * 10^{-4}$ and for $\boldsymbol{R e}(=1,2,4,8)$; A) right-to-left runner movement; B) left-to-right runner movement. 


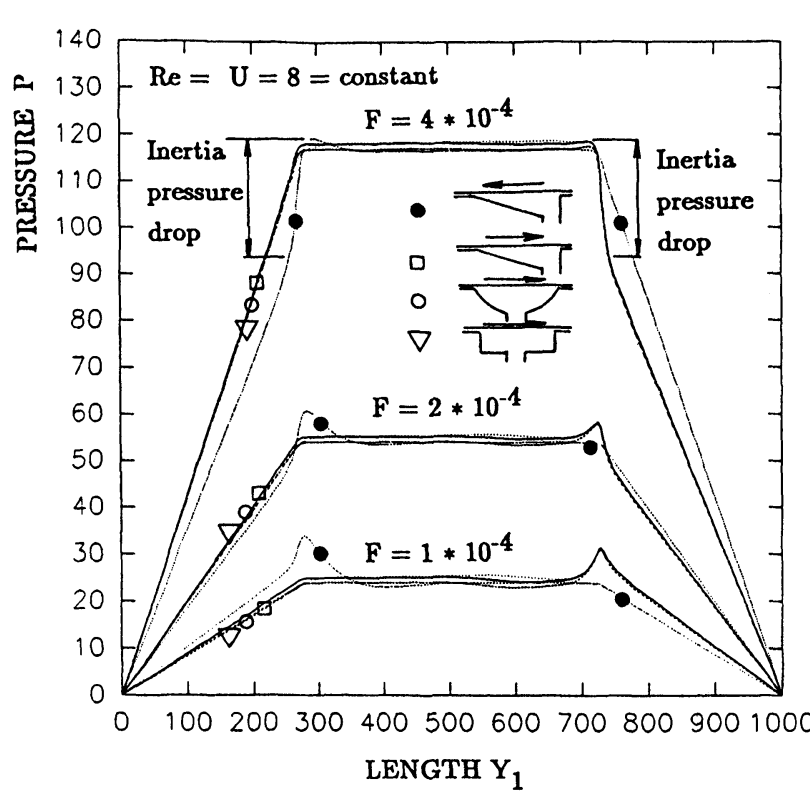

FIGURE 8 Effect of the jet strength $\boldsymbol{F}\left(=1 * 10^{-4}, 2 * 10^{-4}, 4 * 10^{-4}\right)$ on the pressure profiles at constant $\boldsymbol{R} \boldsymbol{e}=8$.

generated by the velocity of the runner or the strength of the jet.

\section{Acknowledgement}

The work of this paper was supported in part by NASA Lewis Research Center under research grant NAG-3-1547. The authors are grateful to Mr. Jim Walker for his support.

\section{Nomenclature}

A

C

D

F

$h$

$J$

$L$

$M$

$n$

$P$

$U_{i}$

$\boldsymbol{U}_{\boldsymbol{i}}$

$\boldsymbol{R e}$

$R_{s}$

$S_{m}$ $x^{j}$
$X^{j}$

$X^{j}$

$y^{j}$

$Y^{j}$

$\beta_{j}^{i}$

$\mu$

р

\section{References}

Artiles, A., Walowit, J., and Shapiro, W., 1982. Analysis of Fluid Film Journal Bearings with Turbulence and Inertia Effects, Advances in Computer Aided Bearing Design, ASME.

Aoyama, T., Inasaki, I., and Yonetsu, S. 1982. Limiting Conditions of Hydrostatic Bearings, Bull. Japan Soc. of Prec. Eng. Vol. 16, no. 2, pp. 84-90.

Belousov, A.I. and Yu, A.R., 1976. Dynamic Characteristics of Liquid Film in Hybrid Hydrostatic Bearing. Soviet Aeronautics, Vol. 21, no. 3, pp. 16-19, Allerton Press.

Betts, C., and Roberts, W.H., 1969. A Theoretical and Experimental Study of a Liquid-Lubricated Hydrostatic Journal Bearing, Proc. IMechE Tribology Group, Vol. 183, pt. 1, No. 32, pp. 647-657.

Bou-Said, B., and Chaomleffel, J.P., 1988. Hybrid Journal Bearings: Theoretical and Experimental Results, 88-Trib-55.

Braun, M.J., and Wheeler, R.L., 1987. A Fully Coupled Variable Properties Thermohydraulic Model for a Cryogenic Hydrostatic Journal Bearing, J. Tribology, Vol. 109, pp. 405-417.

Braun, M.J., 1990. Discussion, Tribology Transactions, Vol. 33, no. 4, pp. 547-550.

Braun, M.J., and Batur, C., 1991. Non-Intrusive Laser Based Full Field Quantitative Flow Measurements Aided by Digital Image Processing, Part 2: The Case of Hydrostatic Bearing, Tribology International, Vol. 24, no. 5, pp. 277-290.

Braun, M.J., Choy, F.K., and Zhou, Y.M., 1993a. The Effects of a Hydrostatic PocketAspect Ratio and its Supply Orrifice Position and Attack Angle on Steady State Flow Patterns, Pressure and Shear Characteristics, ASME Journal of Tribology, Vol. 115, no. 4, pp. 678-685.

Braun, M.J., Zhou, Y.M., and Choy, F.K., 1993b. Transient Flow Patterns and Pressures Characteristics in a Hydrostatic Pocket. Paper accepted for publication in the ASME J. of Tribology.

Brandeis, J., and Rom, J., 1980. Interactive Method for Computation of Viscous Flow with Recirculation, Computational Physics, Vol. 40 pp. 396-410.

Castelli, V., and Shapiro, W., (1967). Improved Methods for Numerical Solutions of the General Fluid Film Lubrication Problem, J. Lubrication Technology, Vol. 89, pp. 71-79.

Chaomleffel, J. P., and Nicholas, D., 1986. Experimental Investigations of Hybrid Journal Bearings, Tribology International, Vol. 19, no.5, pp. 253-259.

Constantinescu, V.N., and Galetuse, S., 1976. Pressure Drop Due to Inertia Forces in Step Bearings, J. Lubrication Technology, Vol. 98, pp. 167-174.

Davies, P. B., and Leonard, R. 1970. The Dynamic Behavior of Multi-Recess Hydrostatic Journal Bearings, IMechE Tribology Group Convention, Vol. 184, P3L, May, pp. 139-147.

Dzodzo M., 1991. Laminar Natural Convection in Some Enclosures of Arbitrary Cross Section, Ph.D Thesis, Faculty of Mechanical Eng., University of Belgrade. 
Ettles, C., 1968. Solutions for Flow in a Bearing Groove, Proc. Inst. of Mech. Eng., Vol. 182, Part 3N, pp. 122-133.

Goryunov, L., and Belousov, A. (1969). Certain Design Features of Radial Hydrostatic Bearings for High-Speed Machines, Izvstiya VUZ. Aviatsionnya Tekhnika, vol. 12, no. 2, pp. 162-168.

Heckelman, D.D., and Ettles, C., 1987. Viscous and Inertial Pressure Effects at the Inlet of a Bearing Film. ASLE Preprint No. 87-AM2A-1.

Heller, S. 1974. Static and Dynamic Performance of Externally Pressurized Fluid Film Journal Bearings in the Turbulent Regime, ASME J. of Lubrication Technology, Vol. 96, July, pp. 381-390.

Ho. Y.S., and Chen, N.N.S., 1979. Performance Characteristics of a Capillary-Compensated Hydrostatic Journal Bearing, Wear, Vol. 52, pp. 285-295.

Ho, Y.S., and Chen, N.N.S., (1980). Dynamic Characteristic of a Hydrostatic Journal Bearing, Wear, Vol. 63, pp. 13-24.

Ho, Y.S., and Chen, N.N.S., 1984. Pressure distribution in a Six Pocket Hydrostatic Bearing, Wear, Vol. 98, pp. 89-100.

Hunt, J.B., and Ahmed, K.M., 1968. Load Capacity, Stiffness and Flow Characteristics of a Hydrostatically Lubricated Six Pocket Journal Bearing Supporting a Rotating Spindle, Proc. IMechE, Vol. 182, P3N, pp. 53-62.

Kumar, R., and Yuan, T.D., 1989. Recirculating Mixed Convection Flows in Rectangular Cavities, J. Thermophysics, Vol. 3, no. 3, pp. 321-329.

Leonard, R., and Davies, P.B. (1971). An Experimental Investigation of the Dynamic Behavior of a Four Recess Hydrostatic Journal Bearing, IMechE Externally Pressurized Bearings, Paper C29, pp. 245-261.

O'Brien, V., 1982. Viscous, Flow in an Annulus With a Sector Cavity, J. Fluids Engineering, Vol. 104, pp. 500-504.

Pan, C.H.T., 1974. Calculation of Pressure, Shear, and Flow in Lubrication Films for High Speed Bearings, J. Lubrication Technology, Vol. 96, pp. 80-94.

Patankar, S.V., and Spalding, D.B., (1972). A Calculation Procedure for Heat, Mass and Momentum Transfer in Three-Dimensional Parabolic Flows, Int. J. Heat Mass Transfer, Vol. 15, p. 1787- 1806.
Patankar, S.V., (1980), Numerical Heat Transfer and Fluid Flow, New York: McGraw-Hill.

Peric, M., (1985). A Finite Volume Method for the Prediction of Three-Dimensional Fluid Flow in Complex Ducts, Ph.D. Thesis, University of London.

Pratap V.S., and Spalding D.B., (1976). Fluid Flow and Heat Transfer in Three-Dimensional Duct Flows, Int. J. Heat Mass Transfer, Vol. 19, pp. 1183-1188.

Redecliffe, J. and Vohr, J. 1969, Hydrostatic Bearings for Cryogenic Rocket Engine Turbopumps, ASME J. Lub. Tech., July, pp. 557-575. San Andres, L.A., 1990. Analysis of Turbulent Hybrid Bearings with Fluid Inertia Effects, J. Tribology, Vol. 112, pp. 699-707.

San Andres, L.A., Velthuis, J.F.M., 1991. Laminar Flow in a Recess of a Hydrostatic Bearing, STLE preprint 91-TC-3B-3, STLE/ASME Tribology Conference, St. Louis, Missouri, October 14-16, 1991.

Scharer, J.K., Tellier, J., and Hibbs, R., 1991a. A Study of the Transient Performance of the Hydrostatic Journal Bearings: Part I-Test Apparatus and Facility, STLE preprint No. 91-TC-3B-1.

Scharer, J.K., Tellier, J., and Hibbs, R., 1991b. A Study of the Transient Performance of the Hydrostatic Journal Bearings: Part II-Experimental Results, STLE preprint No. 91-TC-3B-2.

Shinkle, J., and Hornung, K., 1965. Frictional Characteristics of Liquid Hydrostatic Journal Bearings, ASME J. of Basic Engineering, Ser. D, Vol. 87, pp. 163-169.

Shyy, W., 1985. Numerical Outflow Boundary Condition for NavierStokes Flow Calculations by a Line Iterative Method, AIAA Journal, Vol. 23, no. 12, December, pp. 1847-1848.

Spica, P.W., Hannum, N.P., and Meyer, S.D., 1986. Evaluation of Hybrid Hydrostatic Bearing for Cryogenic Turbopump Application, NASA TM872254.

Tichy, J.A., and Bourgin, P., 1985. The Effect of Inertia in Lubrication Flow Including Entrance and Initial Conditions, J. Appl. Mech., Vol. 52, pp. 759-765.

Zuk, J., and Renkel, H.E., (1971), Numerical Analysis of Flow and Pressure Fields in an Idealized Spiral Grooved Pumping Seal, NASA TN D-6183 

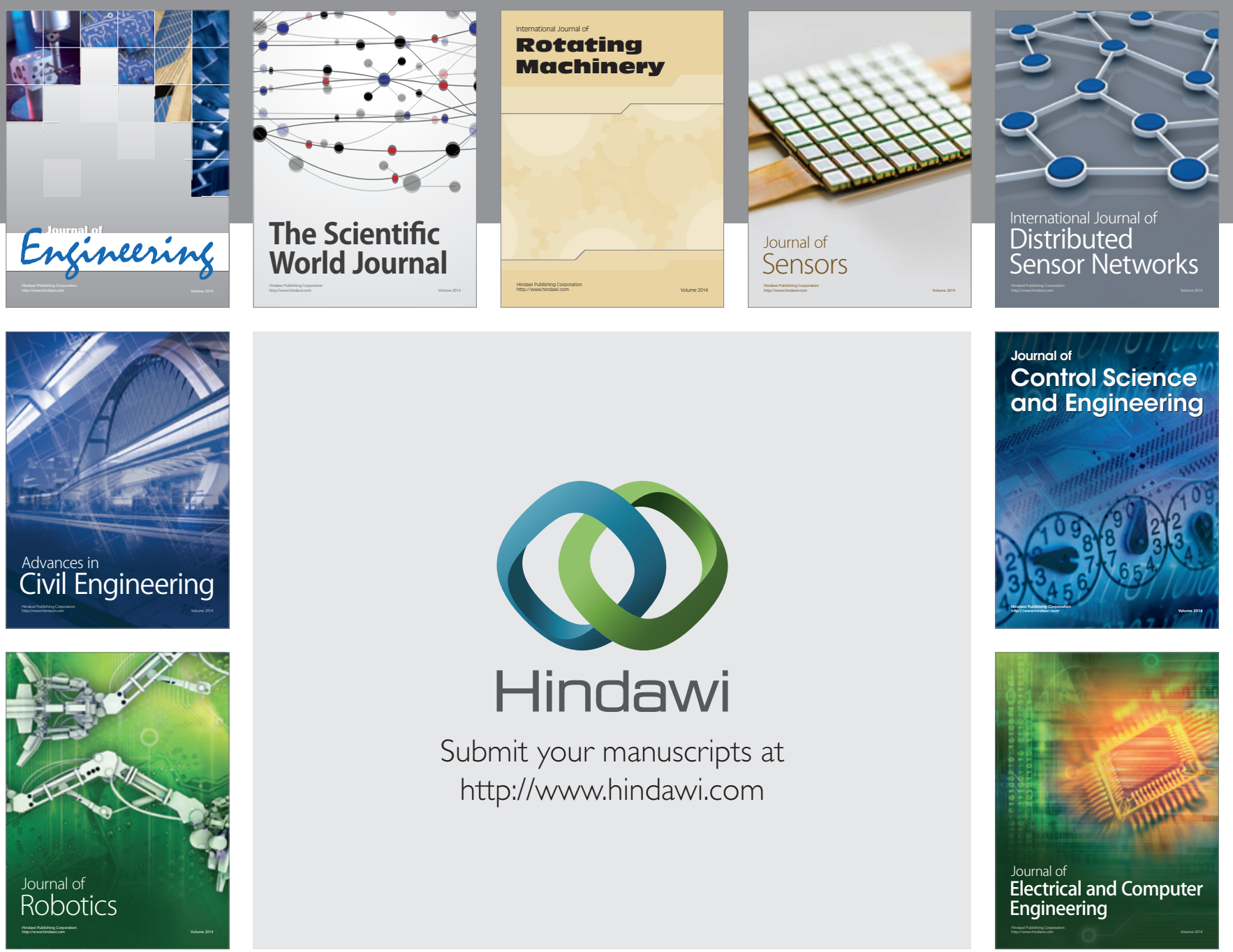

Submit your manuscripts at

http://www.hindawi.com
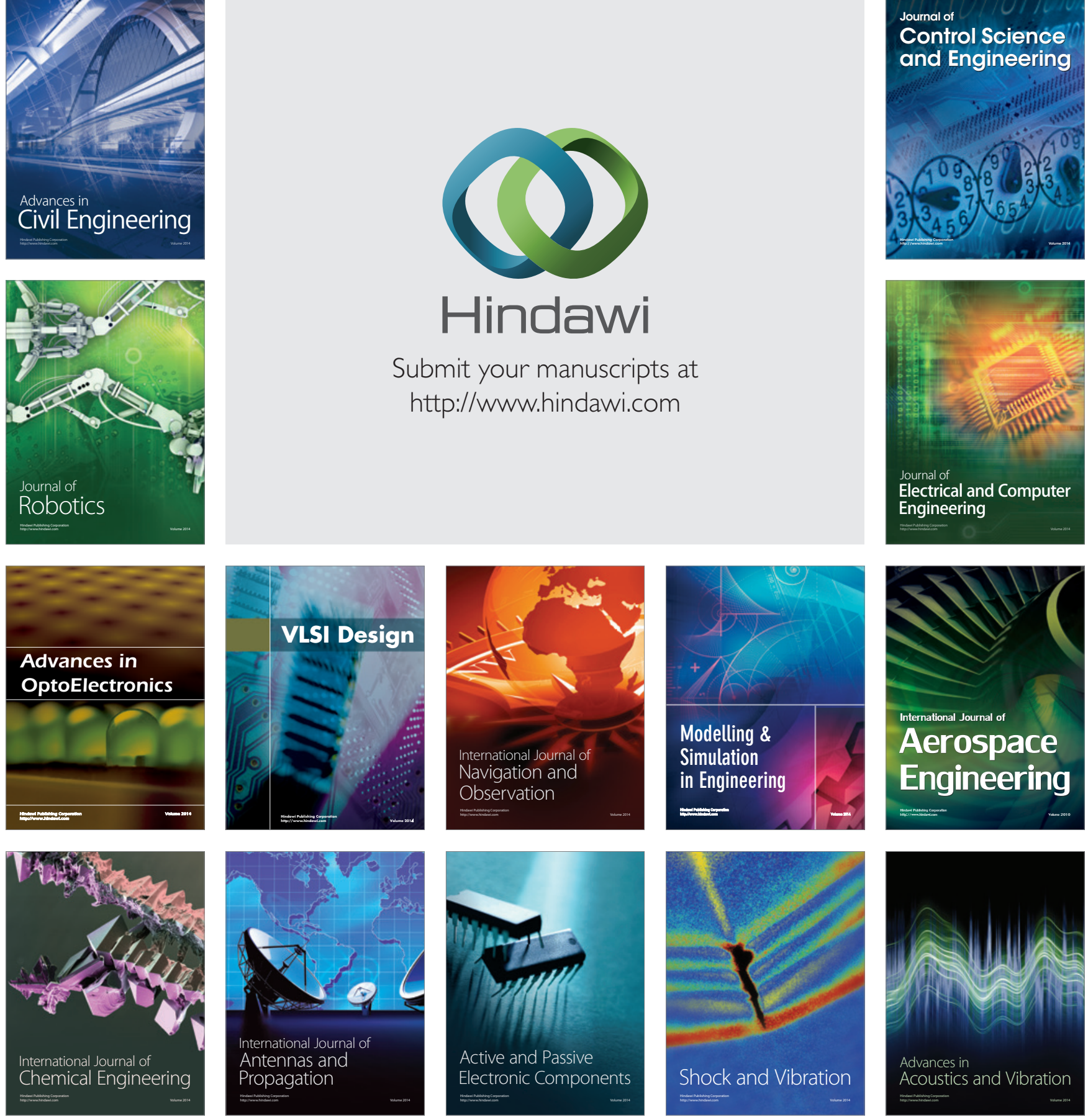